\title{
CORRESPONDENCE
}

\section{Left ventricular overloading is the leading mechanism in extubation failure of patients at high-risk of weaning-induced pulmonary edema}

\author{
Marine Goudelin ${ }^{1,2}$, Bruno Evrard $^{1,2}$ and Philippe Vignon ${ }^{1,2,3,4^{*}}$ (D)
}

๑ 2020 Springer-Verlag GmbH Germany, part of Springer Nature

\section{Dear Editor,}

We read with interest the correspondence by Sanfilippo et al. [1] who suggest performing further analysis for our recently reported study [2]. As per their thoughtful suggestion, we first redefined the two study groups as "weaning success" $(n=43)$ when the patient could be extubated without reinstitution of ventilator support within $48 \mathrm{~h}$ after extubation, and as "weaning failure" $(n=16)$ whenever one the following occurred: (i) failed spontaneous breathing trial (SBT) $(n=12)$; (ii) reintubation and/or resumption of ventilator support within $48 \mathrm{~h}$ after extubation $(n=3)$; or (iii) death in the $48 \mathrm{~h}$ after extubation $(n=1)$ [3]. When compared to patients who succeeded weaning, patients who failed had greater tachycardia and exhibited a higher $\mathrm{E}$ wave maximal velocity $(103 \mathrm{~cm} / \mathrm{s}[84-140]$ vs. $81 \mathrm{~cm} / \mathrm{s}[61-89]: ~ p=0.002)$, an increased E/A ratio (1.4 [0.9-2.3] vs. 0.9 [0.7-1.1]: $p=0.002)$, a shorter $\mathrm{E}$ wave deceleration time $(110 \mathrm{~ms}$ [97-158] vs. $173 \mathrm{~ms}$ [130-212]: $p=0.014)$ and a higher tricuspid regurgitation peak velocity $(3.37 \mathrm{~m} / \mathrm{s}$ [2.703.87 vs. $2.83 \mathrm{~m} / \mathrm{s}$ [2.57-3]: $p=0.034$ ), while baseline left ventricular (LV) ejection fraction and LV outflow tract velocity-time integral were lower (29\% [18-36] vs. $35 \%$ [26-40]: $p=0.056$; and 14.5 [8.5-19.1] vs. 16.7 [13.521.1]: $p=0.034$, respectively). In contrast, no significant difference in the $E^{\prime}$ wave maximal velocity and $E / E^{\prime}$ ratio was noted (Table 1). During SBT, variations of Doppler

\footnotetext{
*Correspondence: philippe.vignon@unilim.fr

${ }^{4}$ Réanimation Polyvalente, CHU Dupuytren, 2 Avenue Martin Luther king, 87042 Limoges, France
}

Full author information is available at the end of the article parameters were more marked in patients who failed than in those who succeeded weaning: higher increase of E wave maximal velocity $(122 \mathrm{~cm} / \mathrm{s}$ [92-160] vs. $93 \mathrm{~cm} / \mathrm{s}$ [73-105]: $p=0.002)$ and of $E / A$ ratio $(1.7$ [1-3.5] vs. 0.9 [0.7-1.3]: $p=0.001)$, and greater shortening of $E$ wave deceleration time (88 $\mathrm{ms}$ [74-141] vs. $147 \mathrm{~ms}$ [105-174]: $p=0.024)$. Again, no significant difference was observed for the $E^{\prime}$ wave maximal velocity and $E / E^{\prime}$ ratio (Table 1 ). These data confirm those reported initially when dichotomizing patients according to passed or failed SBT [2].

Second, we investigated if the grade of LV diastolic dysfunction was associated with weaning failure [4]. Not surprisingly, the proportion of higher grades of LV diastolic dysfunction was significantly higher in patients who failed weaning, both at baseline and during SBT (Table 1). At baseline, grade 1 was more frequent in the "weaning success" group, whereas grades 2 and 3 were more prevalent in the "weaning failure" group (25/43 [58\%] vs. $3 / 16$ [19\%] and $13 / 16$ [81\%] vs. $18 / 43$ [42\%], respectively: $p=0.007)$. This highlights the facilitating role of underlying diastolic dysfunction in precipitating LV overload at the origin of weaning-induced pulmonary edema [5].

Third, we performed a subgroup analysis in patients with chronic obstructive pulmonary disease $(n=15)$. In this subset of patients, those who failed weaning $(n=4)$ tended to have a higher $E$ wave maximal velocity $(119 \mathrm{~cm} / \mathrm{s}$ [58-144] vs. $87 \mathrm{~cm} / \mathrm{s}$ [61-96]: $p=0.177)$ and exhibited increased E/A ratio (1.2 [0.9-3.5] vs. 0.9 [0.71]: $p=0.04)$ at baseline.

Overall, these additional analyses confirm the leading role of LV overload in the weaning failure of patients at high risk of developing a weaning-induced pulmonary 
Table 1 Hemodynamic parameters and echocardiographic findings obtained immediately before and during the first spontaneous breathing trial (SBT) according to weaning success or failure

\section{Before SBT}

Hemodynamic parameters

\begin{tabular}{|c|c|c|c|}
\hline $\mathrm{HR}$, bpm & $90(79-102)$ & $114(100-123)$ & 0.001 \\
\hline $\mathrm{sBP}, \mathrm{mmHg}$ & $142(130-151)$ & $134(130-151)$ & 0.500 \\
\hline $\mathrm{dBP}, \mathrm{mmHg}$ & $78(71-86)$ & $81(71-88)$ & 0.511 \\
\hline $\mathrm{mBP}, \mathrm{mmHg}$ & $97(87-107)$ & $98(85-109)$ & 0.993 \\
\hline \multicolumn{4}{|c|}{ Echocardiography findings } \\
\hline$E, \mathrm{~cm} / \mathrm{s}$ & $81(61-89)$ & $103(84-140)$ & 0.002 \\
\hline$A, \mathrm{~cm} / \mathrm{s}$ & $86(70-106)$ & $63(44-125)$ & 0.290 \\
\hline$E / A$ & $0.9(0.7-1.1)$ & $1.4(0.9-2.3)$ & 0.002 \\
\hline DTE, ms & $173(130-212)$ & 110 (97-158) & 0.014 \\
\hline$E^{\prime}$ lateral, $\mathrm{cm} / \mathrm{s}$ & $9.6(7.4-12.2)$ & $11.1(8.2-15.4)$ & 0.230 \\
\hline$E / E^{\prime}$ & $7.9(5.6-11)$ & $9.2(6.6-12.5)$ & 0.170 \\
\hline MR/LA area & $0.2(0.17-0.25)$ & $0.15(0.13-0.2)$ & 0.170 \\
\hline LVEF, \% & $35(26-40)$ & $29(18-36)$ & 0.056 \\
\hline LVOT VTI, cm & $16.7(13.5-21.1)$ & $14.5(8.5-19.1)$ & 0.069 \\
\hline TR peak velocity, $\mathrm{m} / \mathrm{s}$ & $2.83(2.57-3)$ & $3.37(2.7-3.87)$ & 0.034 \\
\hline \multicolumn{4}{|c|}{ Left ventricular diastolic dysfunction* } \\
\hline Grade 1 & $25(58 \%)$ & $3(19 \%)$ & 0.008 \\
\hline Grade 2 & $16(37 \%)$ & $9(56 \%)$ & \\
\hline Grade 3 & $2(5 \%)$ & $4(25 \%)$ & \\
\hline
\end{tabular}

\section{During SBT}

Hemodynamic parameters

\begin{tabular}{|c|c|c|c|}
\hline $\mathrm{HR}$, bpm & $96(84-110)$ & $121(109-132)$ & 0.002 \\
\hline $\mathrm{sBP}, \mathrm{mmHg}$ & $147(126-156)$ & $143(120-166)$ & 0.946 \\
\hline $\mathrm{dBP}, \mathrm{mmHg}$ & 78 (70-92) & $89(70-98)$ & 0.314 \\
\hline $\mathrm{mBP}, \mathrm{mmHg}$ & $103(90-110)$ & $112(89-116)$ & 0.177 \\
\hline \multicolumn{4}{|c|}{ Echocardiography findings } \\
\hline$E, \mathrm{~cm} / \mathrm{s}$ & $93(73-105)$ & $122(92-160)$ & 0.002 \\
\hline$A, \mathrm{~cm} / \mathrm{s}$ & $86(75-112)$ & $64(33-117)$ & 0.127 \\
\hline$E / A$ & $0.9(0.7-1.3)$ & $1.7(1-3.5)$ & 0.001 \\
\hline DTE, ms & $147(105-174)$ & $88(74-141)$ & 0.024 \\
\hline$E^{\prime}$ lateral, $\mathrm{cm} / \mathrm{s}$ & $9.9(8-11.3)$ & $12.5(8-16.1)$ & 0.079 \\
\hline$E / E^{\prime}$ & $9.5(7.2-12.7)$ & $9.6(7.8-13.5)$ & 0.500 \\
\hline MR/LA area & $0.21(0.16-0.23)$ & $0.26(0.14-0.39)$ & 0.330 \\
\hline LVEF, \% & $33(25-43)$ & $27(15-37)$ & 0.087 \\
\hline LVOT VTI, cm & $17.8(14.4-20.8)$ & $12.9(8.2-18.9)$ & 0.012 \\
\hline TR peak velocity, $\mathrm{m} / \mathrm{s}$ & $3(2.75-3.3)$ & $3.44(3.1-4.16)$ & 0.047 \\
\hline \multicolumn{4}{|c|}{ Left ventricular diastolic dysfunction* } \\
\hline Grade 1 & $19(44 \%)$ & $3(19 \%)$ & 0.007 \\
\hline Grade 2 & $22(51 \%)$ & $6(38 \%)$ & \\
\hline Grade 3 & $2(5 \%)$ & $7(43 \%)$ & \\
\hline
\end{tabular}

$H R$ heart rate, $s B P$ systolic blood pressure, $d B P$ diastolic blood pressure, $m B P$ mean blood pressure, $D T E$ deceleration time of mitral $\mathrm{E}$ wave, $M R$ mitral regurgitation, $L A$ left atrium, LVEF left ventricular ejection fraction, LVOT left ventricular outflow tract, VTI velocity-time integral, $T R$ tricuspid regurgitation

${ }^{*}$ According to [4]

edema. Whether a therapeutic strategy based on such hemodynamic assessment could facilitate weaning in this targeted population remains to be determined. 


\section{Author details}

${ }^{1}$ Medical-Surgical Intensive Care Unit, Dupuytren Teaching Hospital, Limoges, France. ${ }^{2}$ Inserm CIC 1435, Dupuytren Teaching Hospital, Limoges, France.

${ }^{3}$ Faculty of Medicine, University of Limoges, Limoges, France. ${ }^{4}$ Réanimation Polyvalente, CHU Dupuytren, 2 Avenue Martin Luther king, 87042 Limoges, France.

\section{Compliance with ethical standards}

\section{Conflicts of interest}

Authors have no conflicts of interest to declare.

\section{Publisher's Note}

Springer Nature remains neutral with regard to jurisdictional claims in published maps and institutional affiliations.

\section{Accepted: 24 July 2020}

Published online: 6 August 2020

\section{References}

1. Sanfilippo F, Murabito P, La Rosa V, Oliveri F, Astuto M (2020) Successful spontaneous breathing trial, early reintubation and mechanisms of weaning failure. Intensive Care Med. https://doi.org/10.1007/s00134-02006176-2

2. Goudelin M, Champy P, Amiel JB, Evrard B, Fedou AL, Daix T, François B, Vignon $P$ (2020) Left ventricular overloading identified by critical care echocardiography is key in weaning-induced pulmonary edema. Intensive Care Med 46:1371-1381

3. Beduneau G, Pharm T, Schortgen F, Piquilloud L, Zogheib E, Jonas M, Grelon F, Runge I, Nicolas T, Grange S, Barberet G, Guitard PG, Frat JP, Constan A, Chretien JM, Mancebo J, Mercat A, Richard JM, Brochard L (2017) Epidemiology of weaning outcome according to a new definition. The WIND study. Am J Respir Crit Care Med 195:772-783

4. Nagueh SF, Smiseth OA, Appleton CP, Byrd BF 3rd, Dokainish H, Edvardsen T, Flachskampf FA, Gillebert TC, Klein AL, Lancellotti P, Marino P, Oh JK, Alexandru Popescu B, Waggoner AD (2016) Recommendations for the evaluation of left ventricular diastolic function by echocardiography: an update from the american society of echocardiography and the european association of cardiovascular imaging. Eur Heart J Cardiovasc Imaging 17:1321-1360

5. Vignon P (2018) Cardiovascular failure and weaning. Ann Transl Med 6:354 